\title{
FULL AND PARTIAL REGULARITY FOR A CLASS OF NONLINEAR FREE BOUNDARY PROBLEMS
}

\author{
ARAM L. KARAKHANYAN
}

ABStRaCT. In this paper we classify the nonnegative global minimizers of the functional

$$
J_{F}(u)=\int_{\Omega} F\left(|\nabla u|^{2}\right)+\lambda^{2} \chi_{\{u>0\}},
$$

where $F$ satisfies some structural conditions and $\chi_{D}$ is the characteristic function of a set $D \subset \mathbb{R}^{n}$. We compute the second variation of the energy and study the properties of the stability operator. The free boundary $\partial\{u>0\}$ can be seen as a rectifiable $n-1$ varifold. If the free boundary is a Lipschitz multigraph then we show that the first variation of this varifold is bounded and use Allard's monotonicity formula to prove the existence of tangent cones modulo a set of small Hausdorff dimension. In particular we prove that if $n=3$ and the ellipticity constants of the quasilinear elliptic operator generated by $F$ are close to 1 then the conical free boundary must be flat.

\section{INTRODUCTION}

In this paper we consider the regularity of the global minimizers of the functional

$$
J_{F}(u)=\int_{\Omega} F\left(|\nabla u|^{2}\right)+\lambda^{2} \chi_{\{u>0\}} \rightarrow \min
$$

over the class of admissible functions

$$
u \in \mathcal{A}=\left\{u \in W^{1, F}(\Omega), u-u_{0} \in W_{0}^{1, F}(\Omega)\right\}
$$

with $F \in C^{2,1}[0, \infty)$ satisfying the structural conditions

$$
c_{0} \leq F^{\prime}(t) \leq C_{0}, \quad 0 \leq F^{\prime \prime}(t) \leq \frac{C_{0}}{1+t},
$$

for some positive constants $c_{0}, C_{0}$. Here $\Omega \subset \mathbb{R}^{n}$ is a domain, $\lambda>0$ given constant, $W^{1, F}$ the Orlicz-Sobolev space generated by $F$, and $u_{0} \in W^{1, F}(\Omega)$ given Dirichlet datum.

If the free boundary $\partial\{u>0\}$ is smooth then $\nabla u$ satisfies the following implicit Bernoulli type condition

$$
f(\nabla u)+\lambda^{2}-\nabla_{\xi} f(\nabla u) \nabla u=0, \quad f(\xi)=F\left(|\xi|^{2}\right) .
$$

One can deduce that $|\nabla u|=\lambda^{*}$ on the free boundary, where $\lambda^{*}$ is determined from the implicit relation

$$
\lambda^{2}=2 F^{\prime}\left(|\nabla u|^{2}\right)|\nabla u|^{2}-F\left(|\nabla u|^{2}\right) .
$$

Throughout this paper we assume $\lambda^{2}=2 F^{\prime}(1)-F(1)$ so that $|\nabla u|=1$ on $\partial\{u>0\}$, see Remark 4.2 .

2010 Mathematics Subject Classification: 35R35.

Keywords: free boundary, regularity theory, monotonicity formula, free boundary conditions. 
Main Theorem. Let $u \geq 0$ be a global minimizer of (1.1) with F satisfying (1.2).

(a) If $n=3$ and $\partial\{u>0\}$ is a cone and

$$
\sup F^{\prime \prime}\left(|\nabla u|^{2}\right)<\frac{3}{2} F^{\prime}(1)
$$

then $u(x)=\max \left(x_{1}, 0\right)$ in some coordinate system.

(b) Suppose that the rectifiable $n-1$ varifold $V=(\Gamma, \theta)$ associated with $\Gamma=\partial\{u>0\}$ has bounded first variation. If for some $\alpha \in(0,1)$ and $\xi \in \Gamma$

$$
\int_{B_{\rho}(\xi)}|H| \leq C(\xi, \alpha) r^{n-2+\alpha}, \quad r \in(0, R), \overline{B_{R}}(\xi) \subset U \subset \mathbb{R}^{n},
$$

$n=3$ and (1.4) holds then $\Gamma$ is regular at $\xi$. Moreover, the singular set has Hausdorff dimension $\leq 1$.

The proof follows from the combination of Theorems 6.1 and 6.3 proved below. When $F(t)=$ $t^{\frac{p}{2}}, 2<p<5$ then our argument shows that (1.4) is satisfied and the homogeneous free boundaries must be flat in $\mathbb{R}^{3}$. One of the open questions in the free boundary regularity theory asks whether the global minimizers of the Alt-Caffarelli functional [AC81] are flat in $\mathbb{R}^{n}, n \leq 7$. Affirmative answer is given for $n=2$ [AC81], $n=3$ [CJK04], $n=4$ [JS15]. For $n=7$ an analogue of the Simons cone shows that non-flat global minimizers exist for $n \geq 7$ [DSJ09]. Our main results contributes in this direction for nonlinear $F$.

The paper is organized as follows: In section 2 we characterize the flat free boundary points via the variational mean curvature. The free boundary is smooth at the flat points, see Theorem 6.1 [ACF84]. Our argument shows that if the free boundary contains a smooth portion of minimal surface then it should be a plane.

In section 3 we recall some well-known facts about varifolds. The main tool we need to prove our main theorem is the Allard monotonicity formula. For this we need to consider the first variation of the varifold constructed from $\Gamma$, and show that the first variation is finite for Lipschitz multigraphs with nonnegative mean curvature using the weak convergence of mean curvature measure [DTW12]. To every varifold with bounded first variation one can assign a Radon measure which in the case of smooth varifold agrees with the mean curvature vector, which is the trace of the second fundamental form. If the relative density of this measure decays sufficiently fast at some point $\xi$ then it implies existence of tangent cones. For the classical case [AC81] the existence of tangent cones follows from the monotonicity formula of Weiss [Wei98] which is a version of linearized radially symmetric entropy for minimal surfaces in $\mathbb{R}^{n}$.

Section 4 contains the main technical tool to be used in the proof of Main Theorem, the stability inequality (4.1). We follow the argument of [CJK04] closely. Our main task is to obtain the expansion for the gradient term for perturbed function in the functional which in the classical case c easily follows from divergence formula thanks to the fact that the Laplace operator is self-adjoint. We also discuss the related stability operator and provide some explicit computations in section 5

In section 6 we prove our main theorem by combining the results we obtained in previous sections. Lastly, we prove a global result for smooth minimizers in $\mathbb{R}^{2}$ at the end of this section.

Finally, in Section 7 we construct a weak solution to the free boundary problem such that its free boundary is a double cone. For simplicity we consider the case $F(t)=t^{p / 2}$. We prove that this solution is not a minimizer. 
Notation. We fix some notation be used throughout of paper: Let $u$ be a minimizer of (1.1) then $\Omega_{u}^{+}=\Omega^{+}(u)=\{u>0\}, \Omega_{R}^{+}(u)=\Omega^{+}(u) \cap B_{R}$, where $B_{R}$ is the open ball centered at the origin. The free boundary is denoted by $\Gamma=\partial\{u>0\}$, and $\mathscr{H}^{s}$ is the $s$-dimensional Hausdorff measure. $V=v(\Gamma, \theta)$ is the varifold associated with $\Gamma$ and we usually consider the portion of $\Gamma$ in some bounded subdomain $U \subset \mathbb{R}^{n}$ where $0 \in \Gamma \cap U$.

\section{VARiational MEAN CURVATURE}

In this section we characterize the flat free boundary points in terms of small density of variational mean curvature of $\partial\{u>0\}$. For every set $E$ of locally finite perimeter we can find an integrable function $H$ which is the variational mean curvature of $E$ [BGM03].

Lemma 2.1. Let $u_{0}$ be a global minimizer of (1.1) then the free boundary $\partial_{\text {red }}\left\{u_{0}>0\right\}$ is a generalized surface of non-positive outward mean curvature, i.e. if $S \subset \partial_{\text {red }}\left\{u_{0}>0\right\}$ and $S^{\prime} \subset$ $\left\{u_{0}>0\right\}$ such that $\partial S=\partial S^{\prime}$ then

$$
\mathscr{H}^{n-1}(S) \leq \mathscr{H}^{n-1}\left(S^{\prime}\right) .
$$

Proof. Recall that $\partial_{\text {red }}\left\{u_{0}>0\right\}$ is relatively open subset of $\partial\left\{u_{0}>0\right\}$. Moreover, $\partial_{\text {red }}\left\{u_{0}>0\right\}$ is smooth [ACF84]. Consequently in the domain $D$ bounded by $S$ and $S^{\prime}$ we have

$$
0=\int_{D} \mathcal{L} u_{0}=\int_{D} \operatorname{div}\left(2 F^{\prime}\left(\left|\nabla u_{0}\right|^{2}\right) \nabla u_{0}\right) .
$$

After applying the divergence theorem we get that

$$
\lambda^{*} F^{\prime}\left(\left(\lambda^{*}\right)^{2}\right) \mathscr{H}^{n-1}(S)=\int_{S}\left|\nabla u_{0}\right| F^{\prime}\left(\left|\nabla u_{0}\right|^{2}\right)=\int_{S^{\prime}} F^{\prime}\left(\left|\nabla u_{0}\right|^{2}\right) \partial_{\nu} u_{0} .
$$

By Theorem 4.1 [ACF84] we have that $\left|\nabla u_{0}\right| \leq \lambda^{*}$ in $\mathbb{R}^{n}$, thus we conclude from the last identity and (1.2) that

$$
\mathscr{H}^{n-1}(S) \leq \mathscr{H}^{n-1}\left(S^{\prime}\right) .
$$

Suppose that $x_{0} \in S$ and choose the coordinate system near $x_{0}$ so that $x_{n}$ points in the direction of the outer normal to $\left\{u_{0}>0\right\}$ and $x^{\prime}=\left(x_{1}, \ldots, x_{n-1}\right)$. Let $S$ be the graph $x_{n}=f\left(x^{\prime}\right)$ near $x_{0}$, where $f$ is some smooth function over $B_{r}^{n-1}\left(x_{0}\right)=\left\{x^{\prime} \in \mathbb{R}^{n-1}:\left|x^{\prime}-x_{0}^{\prime}\right|<r\right\}$ for some small $r>0$. Then $(2.1)$ can be rewritten in the following equivalent form

$$
\int_{B_{r}^{n-1}\left(x_{0}\right)} \sqrt{1+\left|\nabla_{x^{\prime}} f\right|^{2}} \leq \int_{B_{r}^{n-1}\left(x_{0}\right)} \sqrt{1+\left|\nabla_{x^{\prime}}(f-\varepsilon \phi)\right|^{2}}
$$

for every $0 \leq \phi \in C_{0}^{\infty}\left(B_{r}^{n-1}\left(x_{0}\right)\right)$ and $\varepsilon>0$ small. This implies that

$$
\int_{B_{r}^{n-1}\left(x_{0}\right)} \frac{\nabla_{x^{\prime}} f}{\sqrt{1+\left|\nabla_{x^{\prime}}\right|^{2}}} \nabla \phi \geq 0 .
$$

Therefore $\operatorname{div}_{x^{\prime}}\left(\frac{\nabla_{x^{\prime}} f}{\sqrt{1+\left|\nabla_{x^{\prime}} f\right|^{2}}}\right) \geq 0$ and the mean curvature of $S$ is nonnegative.

For every set of finite perimeter $E \subset \mathbb{R}^{n}$ it is possible to find an integrable function $H$, called the variational mean curvature, such that $E$ minimizes the functional

$$
\mathscr{F}(F, U)=\int_{U}\left|D \chi_{F}\right|+\int_{U} \chi_{F}(x) H(x) d x .
$$

More precisely we have 
Definition 2.2. A set $E$ is said to have variational mean curvature $H$ in $U$ if

(i) $\int_{V}\left|D \chi_{E}\right|<\infty, \forall V \Subset U$,

(ii) $\mathscr{F}(E, U) \leq \mathscr{F}(F, U) \forall V \Subset U, \forall F \subset U$ such that $(E \backslash F) \cup(F \backslash E) \Subset V$.

If $E$ is a set of finite perimeter then we can construct $H$ as follows: take a measurable function $h \geq 0$ such that $\int_{E} h<\infty$ and $\int_{F} h=0$ iff $|F|=0$. For $\sigma \geq 0$ and $F \subset E$, consider the functional

$$
\mathcal{B}_{\sigma}(F)=\int_{\mathbb{R}^{n}}\left|D \chi_{F}\right|+\sigma \int_{E \backslash F} h .
$$

Then for the minimizing sets we have $E_{\sigma} \subset E_{\mu}$ if $0 \leq \sigma<\mu$, and $\cup_{\sigma} E_{\sigma}=E$. By defining

$$
H(x)=-\inf \left\{\sigma h(x), x \in E_{\sigma}, \sigma \geq 0\right\}, \quad \forall x \in E
$$

we obtain the desired variational mean curvature function $H$ defined on $E$. Arguing as above we can define $H$ on $\mathbb{R}^{n} \backslash E$ analogously.

Remark 2.3. The variational mean curvature is not unique. As the construction above shows it depends on the choice of the weight function $h$. An interesting choice is $h(x)=\operatorname{dist}(x, \partial E)$ [ATW93].

Then for given $\sigma$ we have $E_{\sigma}$ with $\partial E_{\sigma}=\Sigma_{r e g}+\Sigma_{s}$ such that $\Sigma_{r e g}$ is $C^{2, \alpha}$ surface and if we denote $M_{\sigma}=\partial E_{\sigma}$ then $H_{\sigma}(x)=\sigma h(x) \nu(x)$ on regular part and

$$
\int_{M_{\sigma}} \operatorname{divX}=-\sigma \int_{M_{\sigma}} h(x) X \cdot \nu
$$

Moreover, as $\sigma \rightarrow \infty$ we have weak converges of measures provided that $\partial E$ is a $C^{1, \alpha}$ graph with nonnegative mean curvature [BGM03].

Next we want to characterize the flat points of $\partial\{u>0\}$ via $H$. Recall that $x_{0} \in \partial\{u>0\}$ is said to be $\varepsilon$ flat in $B_{r}\left(x_{0}\right)$ if

$$
\inf _{\nu}\left\{h: \partial\{u>0\} \cap B_{r}\left(x_{0}\right) \subset S\left(h ; x_{0}, \nu\right) \cap B_{r}\left(x_{0}\right)\right\}<\varepsilon r,
$$

where

$$
\mathcal{S}\left(h ; x_{0}, \nu\right):=\left\{x \in \mathbb{R}^{n}:-h<\left(x-x_{0}\right) \cdot \nu<h\right\}
$$

is the slab of height $2 h$ in unit direction $\nu$. An equivalent form of $(2.3)$ is

$\operatorname{HD}\left(\partial\{u>0\} \cap B_{r}\left(x_{0}\right), \Pi\right)<\varepsilon r \quad$ for some $n-1$ dimensional plane $\Pi$ passing through $x_{0}$, where

is the Hausdorff distance of two sets $A, B$.

$$
\operatorname{HD}(A, B):=\max \left\{\sup _{a \in A} \operatorname{dist}(a, B), \sup _{b \in B} \operatorname{dist}(b, A)\right\}
$$

Theorem 2.4. Let $u$ be a global minimizer of (1.1). Then for every $\varepsilon$ there are $\delta>0, r_{0}>0$ such that if $\frac{1}{r^{n-1}} \int_{B_{r}\left(x_{0}\right)}|H|<\delta, r<r_{0}$ then $B_{r / 2}\left(x_{0}\right) \cap \partial\left\{u_{0}>0\right\}$ is $\varepsilon$ flat.

Proof. Suppose there exists $\varepsilon_{0}>0$ and sequences $\left\{\delta_{k}\right\}_{k=1}^{\infty}, \delta_{k} \rightarrow 0,\left\{r_{k}\right\}_{k=1}^{\infty}, r_{k} \rightarrow 0,\left\{x_{k}\right\}_{k=1}^{\infty}, x_{k} \in$ $\partial\{u>0\}$ such that

$$
\frac{1}{r_{k}^{n-1}} \int_{B_{r_{k}}\left(x_{0}\right)}|H|<\delta_{k}
$$


but

$$
\operatorname{HD}\left(\partial\{u>0\} \cap B_{r_{k}}\left(x_{k}\right), \Pi\right) \geq \varepsilon_{0} r_{k}, \quad \forall n-1 \text { dimensional plane } \Pi \text { passing through } x_{k} .
$$

Let $E_{k}$ be the free boundary of $u_{k}(y)=u\left(x_{k}+r_{k} y\right) / r_{k}$ and set $H_{k}(y)=r_{k} H\left(x_{k}+r_{k} y\right)$. Then we have

$$
\int_{B_{1}}\left|D \chi_{E_{k}}\right|+\int_{B_{1}} H_{k}(y) \chi_{E_{k}} \leq \int_{B_{1}}\left|D \chi_{F}\right|+\int_{B_{1}} H_{k}(y) \chi_{F} \quad\left(E_{k} \backslash F\right) \cup\left(F \backslash E_{k}\right) \Subset V .
$$

Observe that $0 \in E_{k}$ and $\int_{B_{1}}\left|H_{k}\right|<\delta_{k}$. Moreover, there is a subsequence $k_{j} \rightarrow \infty$ such that $u_{k_{j}} \rightarrow$ $u_{0}$ uniformly in $C_{l o c}^{\alpha}, \nabla u_{k_{j}} \rightarrow \nabla u_{0}$ weakly star in $L_{l o c}^{\infty}$ and, moreover, by (3.11) [ACF84] $E_{k_{j}}=$ $\partial\left\{u_{k_{j}}>0\right\}$ converge to $E_{0}=\partial\left\{u_{0}>0\right\}$ in Hausdorff distance. Using a customary compactness argument for BV functions (e.g. Theorem 6.3 [Sim83]) we can extract a subsequence, still labelled $E_{k_{j}}$ such that $\chi_{E_{k_{j}}} \rightarrow \chi_{E_{0}}$ in $B V\left(B_{1}\right)$. It is easy to see that $\left(E_{0} \backslash F\right) \cup\left(F \backslash E_{0}\right) \Subset V$ thanks to the convergence of $E_{k_{j}}$ to $E_{0}$ in Hausdorff distance. Consequently

$$
\int_{B_{1}}\left|D \chi_{E_{0}}\right| \leq \int_{B_{1}}\left|D \chi_{F}\right| \quad\left(E_{0} \backslash F\right) \cup\left(F \backslash E_{0}\right) \Subset V
$$

Therefore $\partial\left\{u_{0}>0\right\}$ is a generalised minimal surface. Note that (2.6) translates to

$$
\operatorname{HD}\left(\partial\left\{u_{0}>0\right\} \cap B_{1}(0), \Pi\right) \geq \varepsilon_{0}, \quad \forall n-1 \text { dimensional plane } \Pi \text { passing through } 0 .
$$

If $u_{0}$ is the limit as above then $\partial\left\{u_{0}>0\right\}$ contains a smooth piece $S$ of a minimal surface, because $\partial_{\text {red }}\left\{u_{0}>0\right\}$ is relatively open in $\partial\left\{u_{0}>0\right\}$, see Theorem 6.2 [ACF84] . Let $e$ be the unit outer normal at some $y_{0} \in S$ such that $y_{0} \in \partial_{\text {red }}\left\{u_{0}>0\right\}$. Introduce $w(x)=1+\partial_{e} u_{0}$, then differentiating $\mathcal{L} u_{0}=0$ in $e$ direction we get that $\operatorname{div}\left(a_{i j} \nabla w\right)=0$, where $a_{i j}=F^{\prime}\left(\left|\nabla u_{0}\right|^{2}\right) \delta_{i j}+2 F^{\prime \prime}\left(\left|\nabla u_{0}\right|^{2} \partial_{i} u_{0} \partial_{j} u_{0}\right)$ is a uniformly elliptic matrix thanks to assumptions (1.2). Since $u_{0}$ is smooth near $y_{0} \in S$ we see that $a_{i j} w_{i j}+b_{i} w_{i}=0$ where $b_{i}=\sum_{j} \partial_{j} a_{i j}$. From Hopf's lemma $\partial_{e} w\left(y_{0}\right) \neq 0$. Choose the coordinate system at $y_{0}$ such that $e$ is pointing in $x_{n}$ direction. From $\left|\nabla u_{0}\right|^{2}=1$ near $y_{0}$ on $S$ we infer that $u_{n} u_{i n}=0$ for every $i=1, \ldots, n-1$. In this coordinate system the mean curvature of $S$ at $y_{0}$ is $\sum_{i=1}^{n-1} \partial_{i i} u_{0}=0$. This in conjunction with the equation $a_{i j} u_{i j}=0$ yields that $u_{e e}\left(y_{0}\right)=$ $u_{n n}\left(y_{0}\right)=0$. However, $\partial_{n} w=u_{n n}=0$ at $y_{0}$ and this is in contradiction with Hopf's lemma. Thus $u_{0}=x_{n}+g\left(x^{\prime}\right), x^{\prime}=\left(x_{1}, \ldots, x_{n-1}\right)$ in $\left\{u_{0}>0\right\}$ for some function $g$. The free boundary condition implies that $|\nabla g|=0$ on $\partial_{\text {red }}\left\{u_{0}>0\right\}$. Since $g$ is continuous it follows that $g$ is constant on $\partial\left\{u_{0}>0\right\}$. Evidently $g$ solves the equation $\mathcal{L} g=F^{\prime}\left(1+|\nabla g|^{2}\right) \Delta g+2 F^{\prime \prime}\left(1+|\nabla g|^{2}\right) \nabla g D^{2} g \nabla g=0$ in $\left\{u_{0}>0\right\}$. Thus without loss of generality we can assume that $g=|\nabla g|=0$ on $\partial\left\{u_{0}>0\right\}$ and $\mathcal{L} g=0$ in $\left\{u_{0}>0\right\}$. Suppose that there is $z_{0} \in \partial_{\text {red }}\left\{u_{0}>0\right\}$ such that $g \geq 0$ (or $g \leq 0$ ) near $z_{0}$. Then Hopf's lemma implies that $g$ vanishes identically. Consequently $g=0$ and $u_{0}=x_{n}^{+}$. But this is in contradiction with (2.7).

Remark 2.5. Let $H \in L^{p}$ be the variational mean curvature in the sense of Definition 2.2. If $p=n$ then the boundary of $E$ is $C^{\alpha}, \alpha \in(0,1)$ away from a set of dimension $n-7$. If $p>n$ then $\partial E$ is almost minimal. If $1 \leq p<n$ we cannot expect any regularity [BGM03].

\section{Mean curvature measure}

In this section we introduce some basic facts about varifolds and show that under some conditions the free boundary $\Gamma$ equipped with $\mathscr{H}^{n-1}$ as weight measure becomes a rectifiable $n-1$ varifold. We also introduce Allard's monotonicity formula [All72] in order to show the existence of tangent cones. 
3.1. First variation of varifold. Recall that by Theorem 6.2 [ACF84] $\Gamma$ is rectifiable. Then we define the rectifiable $n-1$ varifold $V=v(\Gamma, \theta)$ (with multiplicity $\theta$ ) as the equivalence class of all pairs $(\widetilde{\Gamma}, \widetilde{\theta})$ such that $\widetilde{\Gamma}$ is $n-1$ rectifiable, $\mathscr{H}(\Gamma \triangle \widetilde{\Gamma})=0$ and $\theta=\widetilde{\theta}$ a.e. on $\Gamma \cap \widetilde{\Gamma}[\operatorname{Sim} 83]$ page 77 . Then the weight measure is $\mu_{V}=\mathscr{H}^{n-1}\llcorner\theta$. We say that $V$ has bounded first variation if there is a constant $c>0$ such that

$$
\sup _{X \in C_{0}^{1}(U),|X| \leq 1}\left|\int \operatorname{div} X d \mu_{V}\right| \leq c .
$$

By the Riesz represenation theorem there is a vector measure $H$ such that

$$
\int \operatorname{div} X=-\int H \cdot X
$$

Suppose $V$ is smooth then $X=X^{\top}+X^{\perp}$, where $X^{\top}$ and $X^{\perp}$ are the tangential and normal components of $X$, respectively. From the divergence theorem it follows that $\int \operatorname{div} X^{\top}=0$. Writing $X^{\perp}=E n, E=X \cdot n$, we get

$$
\begin{aligned}
\int \operatorname{div} X & =\int \operatorname{div}\left(X^{\perp}\right)=\int \tau_{i} \cdot \nabla_{\tau_{i}}(E n) \\
& =\int \tau_{i} \cdot\left[\nabla_{\tau_{i}} E n+E \nabla_{\tau_{i}} n\right]=\int A\left(\tau_{i}, \tau_{i}\right)(X \cdot n) \\
& =-\int H \cdot X,
\end{aligned}
$$

where $A$ is the second fundamental form, $\tau_{1}, \ldots, \tau_{n-1}$ is any orthonormal basis for the tangent space, and $H$ is the mean curvature measure.

3.2. Lipschitz multigraphs. The mean curvature measure can be prescribed to a class of graphs of semicontinuous functions which are subsolutions to the mean curvature equation in viscosity sense [DTW12]. Suppose that $\left\{f_{\alpha}\right\}$ is a family of Lipschits continuous functions $f_{\alpha}: B_{1}^{\prime} \rightarrow \mathbb{R}, f_{\alpha}(0)=0$ such that $\left|f_{\alpha}\left(x^{\prime}\right)-f\left(y^{\prime}\right)\right| \leq L\left|x^{\prime}-y^{\prime}\right|, x^{\prime}, y^{\prime} \in B_{1}^{\prime}$ for some $L>0$, where $B_{1}^{\prime}=\left\{\left|x^{\prime}\right|<1\right\}, x=\left(x^{\prime}, x_{n}\right)$. Suppose that at $x^{\prime} \in B_{1}^{\prime}$ we have $f_{\alpha}\left(x^{\prime}\right)=f_{\beta}\left(x^{\prime}\right)$ for some $\alpha \neq \beta$. In other words, $\left(x^{\prime}, f_{\alpha}\left(x^{\prime}\right)\right)$ is a point of self intersection of the free boundary. Then the set of such points has zero $n-2$ dimensional Hausdorff measure. To prove the claim it is enough to show that $\left(x^{\prime}, f_{\alpha}\left(x^{\prime}\right)\right) \notin \partial_{\text {red }}\{u>0\}$. Otherwise there is a blow-up $u_{0}$ limit of $u$ at $\left(x^{\prime}, f_{\alpha}\left(x^{\prime}\right)\right)$ such that the free boundary $\partial\left\{u_{0}>0\right\}$ contains a plane, hence it follows from the proof of Theorem 2.4 that at 0 the free boundary $\partial\left\{u_{0}>0\right\}$ cannot have self intersection.

As a corollary we can show that the number of components of $\partial\{u>0\}$ near 0 is bounded: Suppose $\partial\{u>0\} \cap Q=\cup_{\alpha}$ Graph $_{f_{\alpha}}$ where we denote $Q=B_{1}^{\prime} \times[-1,1]$ such that $f_{\alpha}(0)=0$. Then we have from [ACF84] Theorem 3.2 that there is a constant $C$ such that

$$
C \geq \mathscr{H}^{n-1}(\partial\{u>0\} \cap Q) \geq \sum_{\alpha=1}^{N} \int_{B_{1}^{\prime}} \sqrt{1+\left|\nabla_{x^{\prime}} f_{\alpha}\right|^{2}} \geq N \mathscr{H}^{n-2}\left(B_{1}^{\prime}\right) .
$$

Hence $N$ is bounded.

There is a subtle approximation argument [DTW12] Theorem 5.1 and Remark 5.1 that allows to construct an approximating sequence $f_{\alpha}^{k}$ for which $\mathcal{M} f_{\alpha}^{k} \geq \sigma_{k}$ and $\sigma_{k} \rightarrow 0$ boundedly.

Using this argument and weak continuity of the first variations $\delta V_{k}$ for $f_{\alpha}^{k}$ we can show that $V$ has bounded first variation. Indeed, we have 


$$
\begin{aligned}
\left|\int_{\text {Graph }_{f_{\alpha}^{k}}} \operatorname{div} X\right| & =\left|\int_{B_{1}^{\prime}} \sqrt{1+\left|\nabla f_{\alpha}^{k}\right|^{2}} \mathcal{M} f_{\alpha}^{k}\right| X\left|d x^{\prime}\right| \\
& \leq\left|\int_{B_{1}^{\prime}} \sqrt{1+\left|\nabla f_{\alpha}^{k}\right|^{2}}\left(\mathcal{M} f_{\alpha}^{k}-\delta_{k}\right)\right| X\left|d x^{\prime}+\delta_{k} \int_{B_{1}^{\prime}} \sqrt{1+\left|\nabla f_{\alpha}^{k}\right|^{2}}\right| X\left|d x^{\prime}\right| \\
& \leq \sqrt{1+L^{2}} \int_{B_{1}^{\prime}}\left(\mathcal{M} f_{\alpha}^{k}-\delta_{k}\right) d x^{\prime}+\delta_{k} \mathscr{H}^{n-1}(\partial\{u>0\} \cap Q) \\
& =\sqrt{1+L^{2}} \int_{\partial B_{1}^{\prime}} \frac{\nabla_{x^{\prime}} f_{\alpha}^{k} \cdot n^{\prime}}{\sqrt{1+\left|\nabla_{x^{\prime}} f_{\alpha}^{k}\right|^{2}}}-\delta_{k} \mathscr{H}^{n-2}\left(B_{1}^{\prime}\right)+\delta_{k} \mathscr{H}^{n-1}(\partial\{u>0\} \cap Q) .
\end{aligned}
$$

Now the result follows from the lower semicontinuity of the first variations [Sim83] Theorem 40.6.

3.3. Allard's monotonicity formula. We recall Theorem 17.6 from [Sim83]: Let $U \subset \mathbb{R}^{n}$ be a bounded domain and $\xi \in U, 0<\alpha \leq 1, \Lambda \geq 0$ such that $V$ has bounded first variation, the mean curvature $H$ is a $\mu_{V}$ integrable function satisfying

$$
\frac{1}{\alpha} \int_{B_{\rho}(\xi)}|H| \leq \lambda\left(\frac{\rho}{R}\right)^{\alpha-1} \mu\left(B_{\rho}(\xi)\right) \quad \forall \rho \in(0, R)
$$

where $\overline{B_{R}(\xi)} \subset U$, then $f(\rho)=e^{\Lambda R^{1-\alpha} \rho^{\alpha}} \frac{\mu\left(B_{\rho}(\xi)\right)}{\rho^{n-1}}$ is non decreasing function of $\rho$, and in fact

$$
f(\sigma) \leq f(\rho)-\int_{B_{\rho}(\xi) \backslash B_{\sigma}(\xi)} \frac{\left|D^{\perp} r\right|^{2}}{r^{n-1}} .
$$

Furthermore, we have the following

Theorem 3.1. If

$$
\int_{B_{\rho}(\xi)}|H| \leq C(\xi, \alpha) r^{n-2+\alpha}, \quad r \in(0, R), \overline{B_{R}}(\xi) \subset U
$$

then $\Gamma$ has a tangent cone at $\xi$.

The assumption (3.1) implies that the density of $V$ at $\xi$ exists, in view of Theorem 17.7 and Corollary 17.8 [Sim83]. Then the existence of tangent cone follows from Theorem 19.3 [Sim83].

\section{SECOND VARIATION FORMULA}

The second variation of the energy for the classical case $f(t)=t$ has been computed in [CJK04]. Our computation is more involved due to the nonlinear form of $F$. The main result of this section is

Theorem 4.1. Let $u \geq 0$ be a local minimizer of $J_{F}$ in $B_{1}$, and $0 \in \partial\{u>0\}$ such that $\partial\{u>$ $0\} \backslash\{0\}$ is smooth. Then for every $\psi \in C_{0}^{\infty}\left(B_{1} \backslash\{0\}\right)$ there holds

$$
\int_{\Gamma} H \psi^{2} \leq \frac{1}{F^{\prime}(1)} \int_{\Omega^{+}(u)} F^{\prime}\left(|\nabla u|^{2}\right)\left\{|\nabla \psi|^{2}+\frac{2 F^{\prime \prime}\left(|\nabla u|^{2}\right)}{F^{\prime}\left(|\nabla u|^{2}\right)}(\nabla u \nabla \psi)^{2}\right\},
$$

where $H$ is the mean curvature of $\partial\{u>0\}$. 
Remark 4.2. Recall that the free boundary condition has the form

$$
f(\nabla u)+\lambda^{2}-\nabla_{\xi} f(\nabla u) \nabla u=0, \quad f(\xi)=F\left(|\xi|^{2}\right) .
$$

This condition gives that $|\nabla u|=\lambda^{*}$ on the free boundary, where $\lambda^{*}$ is determined from $\lambda^{2}=$ $2 F^{\prime}\left(|\nabla u|^{2}\right)|\nabla u|^{2}-F\left(|\nabla u|^{2}\right)$. We normalize the constant $\lambda^{*}$ such that

$$
|\nabla u|=1 \text { on } \Gamma \text {. }
$$

To do so we take $v=c u$ for a suitable constant $c$ and consequently we see that that $|\nabla v|=1$ on $\Gamma$.

Proof. Take $\varepsilon>0$ and $0 \leq \psi \in C_{0}^{\infty}\left(B_{1} \backslash\{0\}\right)$ and introduce

$$
u_{\varepsilon}=\max (u-\varepsilon \psi, 0)= \begin{cases}u-\varepsilon \psi & \text { if } u>\varepsilon \psi \\ 0 & \text { otherwise. }\end{cases}
$$

We have

$$
\begin{aligned}
\int_{\Omega_{R}^{+}\left(u_{\varepsilon}\right)} f\left(\nabla u_{\varepsilon}\right) & =\int_{\{u>\varepsilon \psi\} \cap B_{R}} f(\nabla(u-\varepsilon \psi)) \\
& =\int_{\Omega_{R}^{+}(u)} f(\nabla(u-\varepsilon \psi))-\int_{\{0<u<\varepsilon \psi\} \cap B_{R}} f(\nabla(u-\varepsilon \psi)) \\
& =I_{1}-I_{2},
\end{aligned}
$$

where

$$
I_{1}=\int_{\Omega_{R}^{+}(u)} f(\nabla(u-\varepsilon \psi)),
$$

and

$$
I_{2}=\int_{\{0<u<\varepsilon \psi\} \cap B_{R}} f(\nabla(u-\varepsilon \psi)) .
$$

We first simplify $I_{1}$. Let us expand $f(\nabla u-\varepsilon \nabla \psi)$ in $\varepsilon$ in order to get

$$
f(\nabla u-\varepsilon \nabla \psi)=A_{0}+\varepsilon A_{1}+\varepsilon^{2} A_{2}+O\left(\varepsilon^{3}\right) .
$$

In fact the coefficients $A_{0}, A_{1}, A_{2}$ can be computed explicitly. Precisely, if $f(\nabla u)=F\left(|\nabla u|^{2}\right)$ then it follows

$$
\begin{aligned}
f(\nabla u-\varepsilon \nabla \psi)= & F\left(|\nabla u|^{2}-2 \varepsilon \nabla u \nabla \psi+\varepsilon^{2}|\nabla \psi|^{2}\right) \\
= & F\left(|\nabla u|^{2}+\varepsilon\left(\varepsilon|\nabla \psi|^{2}-2 \nabla u \nabla u\right)\right) \\
= & F\left(|\nabla u|^{2}\right)+F^{\prime}\left(|\nabla u|^{2}\right) \varepsilon\left(\varepsilon|\nabla \psi|^{2}-2 \nabla u \nabla u\right) \\
& +\frac{F^{\prime \prime}\left(|\nabla u|^{2}\right)}{2} \varepsilon^{2}\left(\varepsilon|\nabla \psi|^{2}-2 \nabla u \nabla u\right)^{2}+O\left(\varepsilon^{3}\right) \\
= & F\left(|\nabla u|^{2}\right)-2 F^{\prime}\left(|\nabla u|^{2}\right) \nabla u \nabla \psi \varepsilon \\
& +\left[F^{\prime}\left(|\nabla u|^{2}\right)|\nabla \psi|^{2}+2 F^{\prime \prime}\left(|\nabla u|^{2}\right)(\nabla u \nabla \psi)^{2}\right] \varepsilon^{2}+O\left(\varepsilon^{3}\right) \\
= & A_{0}+A_{1} \varepsilon+A_{2} \varepsilon+O\left(\varepsilon^{3}\right)
\end{aligned}
$$

where

$$
\begin{aligned}
& A_{0}=F\left(|\nabla u|^{2}\right) \\
& A_{1}=-2 F^{\prime}\left(|\nabla u|^{2}\right) \nabla u \nabla \psi \\
& A_{2}=F^{\prime}\left(|\nabla u|^{2}\right)|\nabla \psi|^{2}+2 F^{\prime \prime}\left(|\nabla u|^{2}\right)(\nabla u \nabla \psi)^{2}
\end{aligned}
$$


Consequently

$$
I_{1}=\int_{\Omega_{R}^{+}(u)} f(\nabla(u-\varepsilon \psi))=\int_{\Omega_{R}^{+}(u)}\left[A_{0}+A_{1} \varepsilon+A_{2} \varepsilon^{2}+O\left(\varepsilon^{3}\right)\right]
$$

Next, we simplify $I_{2}$. We assume that $\partial\{u>0\}$ is parametrized by $z(s), s \in D$ for some domain $D \subset \mathbb{R}^{n-1}$ then we can express the points of $\{0<u<\varepsilon \psi\}$ as $x(s, t)=z(s)-\nu(z(s)) t$ where $\nu(z)$ is the unit outer normal of $\{u>0\}$ at $z$ and $0<t<t_{\varepsilon}(z(s))$. Using (4.6) we obtain

$$
\begin{aligned}
\int_{\{0<u<\varepsilon \psi\} \cap B_{R}} f(\nabla u-\varepsilon \nabla \psi) & =\int_{\{0<u<\varepsilon \psi\} \cap B_{R}}\left(\sum_{i=0}^{2} \varepsilon^{i} A_{i}(x)+O\left(\varepsilon^{3}\right)\right) d x \\
& =\int_{D} \int_{0}^{t_{\varepsilon}(z)}\left(\sum_{i=0}^{2} \varepsilon^{i} A_{i}\left(z+t_{\varepsilon}(y) \nabla u(z)\right)+O\left(\varepsilon^{3}\right)\right)\|\operatorname{det} J(s, t)\| d t d s
\end{aligned}
$$

where $\mathcal{J}(z, t)=\frac{\partial(x)}{\partial(s, t)}$ is the transformation matix. Note that at $s_{0} \in D$ we can rotate the coordinate system such that at $z\left(s_{0}\right)$ we have $\nabla u\left(z\left(s_{0}\right)\right)=-e_{n}$. Then it follows that at $s_{0}$ we have

$$
\begin{aligned}
& \left\|\mathcal{J}\left(s_{0}, t\right)\right\|=\operatorname{det}\left\|\begin{array}{cccc}
z_{s_{1}}^{1}+t u_{1 m} z_{s_{1}}^{m} & z_{s_{2}}^{1}+t u_{1 m} z_{s_{2}}^{m} & \ldots & 0 \\
z_{s_{1}}^{2}+t u_{2 m} z_{s_{1}}^{m} & z_{s_{2}}^{2}+t u_{2 m} z_{s_{2}}^{m} & \ldots & 0 \\
\vdots & \vdots & \ddots & \vdots \\
z_{s_{1}}^{n}+t u_{n m} z_{s_{1}}^{m} & z_{s_{2}}^{n}+t u_{n m} z_{s_{2}}^{m} & \ldots & -1
\end{array}\right\| \\
& =\left\|z_{s_{j}}^{i}\right\|\left\|\left(\delta_{i j}+t D_{s_{i} s_{j}}^{2} u\right)\right\| \quad 1 \leq i, j \leq n-1 \\
& =\left\|z_{s_{j}}^{i}\right\|\left(1+t \sum_{i=1}^{n-1} u_{i i}+O\left(t^{2}\right)\right) \text {. }
\end{aligned}
$$

Recall that [GT01] section 14.6 the mean curvature is

$$
\begin{aligned}
H\left(z\left(s_{0}\right)\right) & =\frac{1}{\left|\nabla u\left(z\left(s_{0}\right)\right)\right|}\left(\Delta u\left(z\left(s_{0}\right)\right)-\frac{\nabla u\left(z\left(s_{0}\right)\right) D^{2} u\left(z\left(s_{0}\right)\right) \nabla u\left(z\left(s_{0}\right)\right)}{\left|\nabla u\left(z\left(s_{0}\right)\right)\right|^{2}}\right) \\
& =\sum_{i=1}^{n-1} u_{i i}\left(z\left(s_{0}\right) .\right.
\end{aligned}
$$

Returning to (4.11) and noting that $\left\|z_{s_{j}}^{i}\right\| d s=d \mathscr{H}^{n-1}(z), 1 \leq i, j \leq n-1$ we get

$$
\int_{\{0<u<\varepsilon \psi\} \cap B_{R}} f(\nabla u-\varepsilon \nabla \psi)=\int_{\Gamma} \int_{0}^{t_{\varepsilon}(z)}\left(\sum_{i=0}^{2} \varepsilon^{i} A_{i}(z+t \nabla u(z))+O\left(\varepsilon^{3}\right)\right)\left(1+t \sum_{i=1}^{n-1} u_{i i}+O\left(t^{2}\right)\right) d t d \mathscr{H}^{n-1} .
$$

From Taylor's formula we have

$$
A_{i}(z+t \nabla u(z))=A_{i}(z)+t \nabla A_{i}(z) \nabla u(z)+\frac{t^{2}}{2}\left(\nabla^{2} A_{i}(z) \nabla u(z)\right) \nabla u(z)+O\left(t^{3}\right) .
$$

Let $H$ be the mean curvature of $\Gamma$ and introduce

$$
I_{2 i}=\int_{\{0<u<\varepsilon \psi\} \cap B_{R}} A_{i}, \quad i=0,1,2
$$


so that

$$
I_{2}=\sum_{i=0}^{2} \varepsilon^{i+1} I_{2 i}
$$

We have from above computations

$$
\begin{aligned}
I_{2 i} & =\int_{\Gamma} \int_{0}^{t_{\varepsilon}}\left\{A_{i}(z)+t \nabla A_{i}(z) \nabla u(z)+\frac{t^{2}}{2}\left(\nabla^{2} A_{i}(z) \nabla u(z)\right) \nabla u(z)+O\left(t^{3}\right)\right\}\left[1+H t+O\left(t^{2}\right)\right] d t d \mathscr{H}^{n-1} \\
& =\int_{\Gamma} A_{i}\left(t_{\varepsilon}+\frac{H}{2} t_{\varepsilon}^{2}+O\left(t_{\varepsilon}^{3}\right)\right)+\int_{\Gamma} \nabla A_{i} \nabla u\left(\frac{t_{\varepsilon}^{2}}{2}+H \frac{t_{\varepsilon}^{3}}{3}+O\left(t_{\varepsilon}^{4}\right)\right)
\end{aligned}
$$

Note that $u\left(z+t_{\varepsilon} \nabla u(z)\right)=\varepsilon \psi\left(z+t_{\varepsilon} \nabla u(z)\right)$ on $\partial\{u>\varepsilon \psi\}$, hence taking Taylor's expansion we obtain

$$
t_{\varepsilon}=\varepsilon \psi+\varepsilon^{2}\left(-\psi^{2} \frac{u_{\nu \nu}}{2}-\psi \psi_{\nu}\right)+O\left(\varepsilon^{3}\right)
$$

From here

$$
\begin{aligned}
I_{2 i}= & \int_{\Gamma} A_{i} \varepsilon \psi+\int_{\Gamma} A_{i}\left[\frac{H}{2} \varepsilon^{2} \psi^{2}+\varepsilon^{2}\left(-\psi^{2} \frac{u_{\nu \nu}}{2}-\psi \psi_{\nu}\right)\right] \\
& +\int_{\Gamma} \nabla A_{i} \nabla u \frac{\varepsilon^{2} \psi^{2}}{2}+O\left(\varepsilon^{3}\right)
\end{aligned}
$$

Denoting $\widetilde{H}=\frac{1}{2}\left(H-u_{\nu \nu}\right)$ we further simplify

$$
\begin{aligned}
I_{2}= & \sum_{i=0}^{2} \varepsilon^{i+1} \int_{\Gamma}\left\{A_{i} \psi+\varepsilon A_{i}\left[\widetilde{H} \psi^{2}-\psi \psi_{\nu}\right]+\varepsilon \nabla A_{i} \nabla u \frac{\psi^{2}}{2}\right\}+O\left(\varepsilon^{3}\right) \\
= & \varepsilon \int_{\Gamma}\left\{A_{0} \psi+\varepsilon A_{0}\left[\widetilde{H} \psi^{2}-\psi \psi_{\nu}\right]+\varepsilon \nabla A_{0} \nabla u \frac{\psi^{2}}{2}\right\} \\
& -\varepsilon^{2} \int_{\Gamma}\left\{A_{1} \psi+\varepsilon A_{1}\left[\widetilde{H} \psi^{2}-\psi \psi_{\nu}\right]+\varepsilon \nabla A_{1} \nabla u \frac{\psi^{2}}{2}\right\}+O\left(\varepsilon^{3}\right) \\
= & \varepsilon \int_{\Gamma} A_{0} \psi-\varepsilon^{2} \int_{\Gamma} A_{1} \psi+A_{0}\left[\widetilde{H} \psi^{2}-\psi \psi_{\nu}\right]+\nabla A_{0} \nabla u \frac{\psi^{2}}{2}+O\left(\varepsilon^{3}\right) .
\end{aligned}
$$

Plugging (4.10) and (4.15) into (4.5) we get the formula

$$
\int_{\Omega_{R}^{+}\left(u_{\varepsilon}\right)} f\left(\nabla u_{\varepsilon}\right)=I_{1}-I_{2} .
$$

From (4.12), (4.13) and (4.14) we get

$$
\begin{aligned}
\left|\{0<u<\varepsilon \psi\} \cap B_{R}\right| & =\int_{\Gamma} \int_{0}^{t_{\varepsilon}}\left(1+H t+O\left(t^{2}\right)\right) d t d \mathscr{H}^{n-1}=\int_{\Gamma}\left(t_{\varepsilon}+H \frac{t_{\varepsilon}^{2}}{2}+O\left(t_{\varepsilon}^{3}\right)\right) d \mathscr{H}^{n-1} \\
& =\int_{\Gamma}\left(\varepsilon \psi+\varepsilon^{2}\left(-\psi^{2} \frac{u_{\nu \nu}}{2}-\psi \psi_{\nu}\right)+\varepsilon^{2} \frac{H}{2} \psi^{2}\right)+O\left(\varepsilon^{3}\right) \\
& =\int_{\Gamma} \varepsilon \psi+\varepsilon^{2}\left(\widetilde{H} \psi^{2}-\psi \psi_{\nu}\right)+O\left(\varepsilon^{3}\right) .
\end{aligned}
$$

Now the comparison of energies yields 


$$
\begin{array}{cl}
0 \geq J_{F}(u)-J_{F}\left(u_{\varepsilon}\right) \stackrel{(4.10)}{=} & \int_{B_{R}} F\left(|\nabla u|^{2}\right)+\lambda^{2} \chi_{\{u>0\}}-\int_{\Omega_{R}^{+}\left(u_{\varepsilon}\right)} F\left(\left|\nabla u_{\varepsilon}\right|^{2}\right)-\lambda^{2} \int_{B_{R}} \chi_{\{u-\varepsilon \psi>0\}} \\
\stackrel{(4.16)}{=} & \int_{B_{R}} F\left(|\nabla u|^{2}\right)-I_{1} \\
& +I_{2}+\lambda^{2} \int_{B_{R}} \chi_{\{0<u<\varepsilon \psi\}} \\
& \stackrel{(4.17)}{=}-\varepsilon \int_{\Omega_{R}^{+}(u)} A_{1}-\varepsilon^{2} \int_{\Omega_{R}^{+}(u)} A_{2}+ \\
& +\varepsilon \int_{\Gamma} A_{0} \psi+\varepsilon^{2} \int_{\Gamma} A_{1} \psi+A_{0}\left(\widetilde{H} \psi^{2}-\psi \psi_{\nu}\right)+\nabla A_{0} \nabla u \frac{\psi^{2}}{2} \\
& +\lambda^{2} \int_{\Gamma} \varepsilon \psi+\varepsilon^{2}\left(\widetilde{H} \psi^{2}-\psi \psi_{\nu}\right)+O\left(\varepsilon^{3}\right) .
\end{array}
$$

Recall the free boundary condition $(4.2)$

$$
\lambda^{2}+f(\nabla u)-\nabla_{\xi} f \nabla u=0,
$$

hence it follows from the divergence theorem and (4.7)-(4.8) that the coefficient of $\varepsilon$ in the expression above vanishes. On the other hand we see that the coefficient of $\varepsilon^{2}$ in (4.18) is

$$
-\int_{\Omega_{R}^{+}(u)} A_{2}+\int_{\Gamma} A_{1} \psi+A_{0}\left(\widetilde{H} \psi^{2}-\psi \psi_{\nu}\right)+\nabla A_{0} \nabla u \frac{\psi^{2}}{2}+\lambda^{2} \int_{\Gamma}\left(\widetilde{H} \psi^{2}-\psi \psi_{\nu}\right) .
$$

Thus letting $\varepsilon \rightarrow 0$ we obtain the inequality

$$
\int_{\Gamma} A_{1} \psi+A_{0}\left(\widetilde{H} \psi^{2}-\psi \psi_{\nu}\right)+\nabla A_{0} \nabla u \frac{\psi^{2}}{2}+\lambda^{2} \int_{\Gamma}\left(\widetilde{H} \psi^{2}-\psi \psi_{\nu}\right) \leq \int_{\Omega_{R}^{+}(u)} A_{2} .
$$

From (4.9) we have that

$$
\begin{aligned}
\int_{\Omega_{R}^{+}(u)} A_{2} & =\int_{\Omega_{R}^{+}(u)} F^{\prime}\left(|\nabla u|^{2}\right)|\nabla \psi|^{2}+2 F^{\prime \prime}\left(|\nabla u|^{2}\right)(\nabla u \nabla \psi)^{2} \\
& =\int_{\Omega_{R}^{+}(u)} F^{\prime}\left(|\nabla u|^{2}\right)\left\{|\nabla \psi|^{2}+\frac{2 F^{\prime \prime}\left(|\nabla u|^{2}\right)}{F^{\prime}\left(|\nabla u|^{2}\right)}(\nabla u \nabla \psi)^{2}\right\}
\end{aligned}
$$

Denoting

$$
I_{3}=\int_{\Gamma} A_{1} \psi+A_{0}\left(\widetilde{H} \psi^{2}-\psi \psi_{\nu}\right)+\nabla A_{0} \nabla u \frac{\psi^{2}}{2}+\lambda^{2} \int_{\Gamma}\left(\widetilde{H} \psi^{2}-\psi \psi_{\nu}\right)
$$

and recalling (4.7),(4.8) we get

$$
\begin{aligned}
I_{3} \stackrel{(4.7),(4.8)}{=} & \int_{\Gamma}-2 F^{\prime} \nabla u \nabla \psi \psi-F \psi \psi_{\nu}-\lambda^{2} \psi \psi_{\nu} \\
& +\int_{\Gamma}\left[F \widetilde{H}+\lambda^{2} \widetilde{H}+F^{\prime} \nabla u D^{2} u \nabla u\right] \psi^{2} \\
= & \int_{\Gamma}\left(2 F^{\prime}-F-\lambda^{2}\right) \psi_{\nu} \psi \\
& +\int_{\Gamma}\left[F \widetilde{H}+\lambda^{2} \widetilde{H}+F^{\prime} \nabla u D^{2} u \nabla u\right] \psi^{2}
\end{aligned}
$$




$$
\begin{array}{ll}
\stackrel{(4.2),(4.13)}{=} & \int_{\Gamma}\left[F \widetilde{H}+\lambda^{2} \widetilde{H}+F^{\prime} u_{\nu \nu}\right] \psi^{2} \\
\stackrel{(4.2)}{=} & \int_{\Gamma}\left[2 F^{\prime} \widetilde{H}+F^{\prime} u_{\nu \nu}\right] \psi^{2} \\
= & \int_{\Gamma}\left[F^{\prime}\left(H-u_{\nu \nu}\right)+F^{\prime} u_{\nu \nu}\right] \psi^{2} \\
= & \int_{\Gamma} F^{\prime} H \psi^{2} \\
= & F^{\prime}(1) \int_{\Gamma} H \psi^{2} .
\end{array}
$$

Combining this with (4.20) and (4.19) we obtain (4.1).

\section{Stability Operator}

Let $a_{i j}$ be the matrix

$$
a_{i j}=\frac{F^{\prime}\left(|\nabla u|^{2}\right)}{F^{\prime}(1)}\left\{\delta_{i j}+\frac{2 F^{\prime \prime}\left(|\nabla u|^{2}\right)}{F^{\prime}\left(|\nabla u|^{2}\right)} u_{i} u_{j}\right\}
$$

and define the operator

$$
L[\psi]=\operatorname{div}(a \nabla \psi)
$$

Then (4.1) tells us that

$$
\int_{\Omega^{+}(u)}(L[\psi]+H \psi) \psi \leq 0, \quad \forall \psi \in C_{0}^{\infty}\left(B_{1} \backslash\{0\}\right) .
$$

Thus $L[\psi]+H \psi$ is the stability operator for the minimization problem.

We can make our computation more explicit. For instance if $f(\xi)=|\xi|^{p}, 2<p<\infty$ then

$$
\begin{aligned}
|\nabla(u-\varepsilon \psi)|^{p}= & \left(|\nabla u|^{2}-2 \varepsilon \nabla u \nabla \psi+\varepsilon^{2}|\nabla \psi|^{2}\right)^{p / 2} \\
= & \left(|\nabla u|^{2}+\varepsilon\left(\varepsilon|\nabla \psi|^{2}-2 \nabla u \nabla \psi\right)\right)^{p / 2} \\
= & |\nabla u|^{p}+\frac{p}{2}|\nabla u|^{p-2} \varepsilon\left(\varepsilon|\nabla \psi|^{2}-2 \nabla u \nabla \psi\right) \\
& +\frac{1}{2} \frac{p}{2}\left(\frac{p}{2}-1\right)|\nabla u|^{p-4} \varepsilon^{2}\left[\varepsilon|\nabla \psi|^{2}-2 \nabla u \nabla \psi\right]^{2}+O\left(\varepsilon^{3}\right) \\
= & |\nabla u|^{p}-\varepsilon p|\nabla u|^{p-2} \nabla u \nabla \psi+\varepsilon^{2}\left[\frac{p}{2}|\nabla u|^{p-2}|\nabla \psi|^{2}+4 \frac{p}{4}\left(\frac{p}{2}-1\right)(\nabla u \nabla \psi)^{2}|\nabla u|^{p-4}\right]+O\left(\varepsilon^{3}\right) \\
= & |\nabla u|^{p}-\varepsilon p|\nabla u|^{p-2} \nabla u \nabla \psi+\varepsilon^{2}\left[\frac{p}{2}|\nabla u|^{p-2}|\nabla \psi|^{2}+\frac{p}{2}(p-2)(\nabla u \nabla \psi)^{2}|\nabla u|^{p-4}\right]+O\left(\varepsilon^{3}\right) \\
= & A_{0}+A_{1} \varepsilon+A_{2} \varepsilon^{2}+O\left(\varepsilon^{3}\right),
\end{aligned}
$$

where

$$
\begin{aligned}
& A_{0}=|\nabla u|^{p} \\
& A_{1}=-p|\nabla u|^{p-2} \nabla u \nabla \psi \\
& A_{2}=\frac{p}{2}|\nabla u|^{p-2}|\nabla \psi|^{2}+\frac{p}{2}(p-2)(\nabla u \nabla \psi)^{2}|\nabla u|^{p-4} .
\end{aligned}
$$


Splitting the integral as in (4.5) we get that

$$
\begin{aligned}
\int_{\Omega_{R}^{+}\left(u_{\varepsilon}\right)}\left|\nabla u_{\varepsilon}\right|^{p} & =\int_{\{u>\varepsilon \psi\} \cap B_{R}}|\nabla(u-\varepsilon \psi)|^{p} \\
& =\int_{\Omega_{R}^{+}(u)}|\nabla(u-\varepsilon \psi)|^{p}-\int_{\{0<u<\varepsilon \psi\} \cap B_{R}}|\nabla(u-\varepsilon \psi)|^{p}
\end{aligned}
$$

and we can carry over the computation of previous section. In this case (4.1) takes form

$$
\int_{\Gamma} H \psi^{2} \leq \int_{\Omega^{+}(u)}|\nabla u|^{p-2}\left\{|\nabla \psi|^{2}+(p-2) \frac{(\nabla u \nabla \psi)^{2}}{|\nabla u|^{2}}\right\} .
$$

\section{Proof of MAIN THEOREM}

First we prove part (a) of Main Theorem.

Theorem 6.1. Let $u \geq 0$ be a homogeneous global solution then $\partial\{u>0\}$ consists of finitely many convex cones. Moreover, if $\sup F^{\prime \prime}\left(|\nabla u|^{2}\right)<\frac{3}{2} F^{\prime}(1)$ then $u$ is a half-plane solution.

Proof. First we estimate the geodesic curvature of $\partial\{u>0\}$. Let us choose $g, h \in C^{\infty}[0, \infty) g, h \geq 0$ where

$$
g(r)= \begin{cases}1 & \text { if } 0 \leq r \leq \frac{1}{2} \\ 0 & \text { if } r \geq \frac{3}{4}\end{cases}
$$

and

$$
h(r)= \begin{cases}1 & \text { if } 0 \leq r \leq 1 \\ 0 & \text { if } r \geq 2\end{cases}
$$

Let us take $\psi_{\varepsilon}(r)=h(r / \varepsilon) g(r) r^{-1 / 2}$, where $\varepsilon>0$ is small, and compute the integrals in the inequality (4.1) for this choice of the test function. We have

$$
\int_{\gamma} \int_{0}^{\infty} \kappa(\alpha(s))\left[\psi_{\varepsilon}(r)\right]^{2} d r d s \leq \widehat{C} \int_{0}^{+\infty}\left[\psi_{\varepsilon}^{\prime}(r)\right]^{2} r^{2} d r \mathscr{H}^{2}\left(\{u>0\} \cap \mathbb{S}^{2}\right)
$$

where $\gamma=\partial\{u>0\} \cap \mathbb{S}^{2}, \widehat{C}=1+2 \sup \frac{F^{\prime \prime}\left(\left|\nabla u_{0}\right|^{2}\right)}{F^{\prime}(1)}$, and $\kappa$ is the geodesic curvature of $\gamma$. Observe that

$$
\begin{aligned}
\int_{0}^{+\infty}\left[\psi_{\varepsilon}^{\prime}(r)\right]^{2} r^{2} d r & =\int_{0}^{+\infty}\left[-\frac{1}{2} r^{-3 / 2} h(r / \varepsilon) g(r)\right]^{2} r^{2} d r+O(1) \\
& =\frac{1}{4} \int_{0}^{+\infty}\left[r^{-1 / 2} h(r / \varepsilon) g(r)\right]^{2} d r+O(1) \\
& =\frac{1}{4} \int_{0}^{+\infty}\left[\psi_{\varepsilon}(r)\right]^{2} d r+O(1)
\end{aligned}
$$

On the other hand

$$
\int_{0}^{+\infty}\left[\psi_{\varepsilon}(r)\right]^{2} d r=c \log 1 / \varepsilon+O(1)
$$

for some $c>0$ hence 


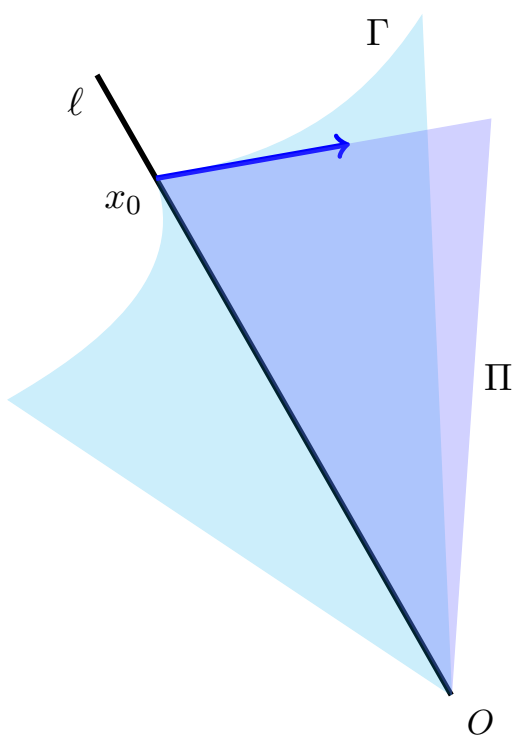

Figure 1. Possible singularity at $x_{0}$.

$$
\lim _{\varepsilon \rightarrow 0} \frac{\int_{0}^{+\infty}\left[\psi_{\varepsilon}^{\prime}(r)\right]^{2} r^{2} d r}{\int_{0}^{+\infty}\left[\psi_{\varepsilon}(r)\right]^{2} d r}=\frac{1}{4}
$$

Consequently we get

$$
\int k d s \leq \frac{\widehat{C}}{4} \mathscr{H}^{2}\left(\{u>0\} \cap \mathbb{S}^{2}\right) .
$$

We observe that the free boundary is smooth away from the vertex. Indeed, from (2.1) we know that the free boundary components are convex cones and suppose there is a ray $\ell$ such that $\Gamma$ has singularity along $\ell$, see Figure 1. If we blow-up $u$ at some $x_{0} \in \ell \backslash\{0\}$ then the free boundary of the blow-up limit (which exists thanks to the compactness of blow-up sequences $u_{j}\left(x_{0}+r_{j}\right) / r_{j}, r_{j} \rightarrow 0$ see section 3 [ACF84]) will contain a flat portion $\Pi$. Then as in the proof of Theorem 2.4 we infer that the blow-up must have smooth free boundary which is a contradiction.

Now we apply the Gauss-Bonnet theorem, [O'N66] page 375, to infer

$$
\mathscr{H}^{2}\left(V_{i}\right)+\int_{\gamma_{i}} \kappa=2 \pi
$$

where $V_{i}$ is the component of $\mathbb{S}^{2} \cap\{u=0\}$ and $\kappa$ is the curvature of $\gamma_{i}=\partial V_{i}$. From here we obtain

or

$$
4 \pi-\mathscr{H}^{2}\left(\mathbb{S}^{2} \cap \Omega^{+}\right)=2 \pi m-\int_{\Gamma \cap \mathbb{S}^{2}} \kappa
$$

$$
2 \pi(m-2)+\mathscr{H}^{2}\left(\mathbb{S}^{2} \cap \Omega^{+}\right)=\int_{\Gamma \cap \mathbb{S}^{2}} \kappa \leq \frac{\widehat{C}}{4} \mathscr{H}^{2}\left(\{u>0\} \cap \mathbb{S}^{2}\right),
$$

which yields the estimate

$$
2 \pi(m-2) \leq\left(\frac{\widehat{C}}{4}-1\right) \mathscr{H}^{2}\left(\{u>0\} \cap \mathbb{S}^{2}\right) .
$$


Clearly if $\widehat{C}<4$ then $m=0,1$.

Consequently the free boundary is Lipschitz graph near 0 . Since every minimizer is also a viscosity solution [DK18] then applying the $C^{1, \alpha}$ regularity result for Lipschitz graphs [Fel01] we conclude that $\Gamma$ cannot have singularity at 0 .

Remark 6.2. For $F(t)=t^{\frac{p}{2}}$ we have that $\widehat{C}=p-1$ hence for

$$
2<p<5, \quad n=3
$$

the homogeneous free boundary is flat.

6.1. Partial regularity. Now we prove part (b) of Main Theorem.

Theorem 6.3. Suppose $V=v(\Gamma, \theta)$ has bounded first variation. If $n=3$ and (1.4) is satisfied then $\Gamma$ is smooth away from a set $\Sigma_{0}$ of singular points and $\operatorname{dim}\left(\Sigma_{0}\right) \leq 1$.

Proof. Let $\alpha \in(0,1)$ and $\varepsilon^{*}>0$ be fixed. Let $E\left(\alpha, \varepsilon^{*}\right)$ be the set of the points $x \in \Gamma$ where

$$
\frac{1}{r^{n-1}} \int_{B_{r}(x)} H d \mu_{V} \geq \varepsilon^{*} r^{\alpha-1} \quad \text { whenever } r<r_{x}
$$

for some small $r_{x}>0$ depending on $x$. Applying Theorem 3.1 we see that $\Gamma$ at $x$ has tangent cone. Then Theorem 6.1 implies that that $\Gamma$ is smooth at $x$ if $n=3$. Hence $E\left(\alpha, \varepsilon^{*}\right) \subset \partial_{\text {red }}\{u>0\}$ if $n=3$.

Suppose that $y \in \Gamma \backslash F\left(\alpha, \varepsilon^{*}\right)$. Then we can choose balls $B_{i}$ with following properties; $\Gamma \backslash$ $F\left(\alpha, \varepsilon^{*}\right) \subset \cup_{i} B_{i}, \operatorname{diam} B_{i} \leq \delta$ for some small $\delta>0$ and

$$
\int_{B_{i} \cap \Gamma} H d \mu_{V} \geq \varepsilon^{*}\left(\operatorname{diam} B_{i}\right)^{n-2+\alpha} .
$$

Then using Vitali's covering theorem Theorem 3.3 [Sim83] we see that the $\delta$ Hausdorff premeasure satisfies the estimate

$$
\mathscr{H}_{\delta}^{1+\alpha}\left((\Gamma \cap U) \backslash E\left(\alpha, \varepsilon^{*}\right)\right) \leq \int_{(\Gamma \cap U) \backslash E\left(\alpha, \varepsilon^{*}\right)} H d \mu_{V} .
$$

Sending $\delta \rightarrow 0$ and recalling that $\alpha<1$ we conclude that $\mathscr{H}^{1+\alpha}\left((\Gamma \cap U) \backslash E\left(\alpha, \varepsilon^{*}\right)\right)=0$. Since the singular points must be in the complement of $E\left(\alpha, \varepsilon^{*}\right)$ then it follows that $\operatorname{dim}(\Sigma) \leq 1$.

6.2. The smooth free boundaries in $\mathbb{R}^{2}$ are lines. Our last result is a simple consequence from the stability inequality (4.1) in $\mathbb{R}^{2}$ where one can use the trick of logarithmic test function.

Theorem 6.4. Suppose that $u$ is a global minimizer of the energy (1.1) in $\mathbb{R}^{2}$ such that the free boundary of $u$ is smooth. Then $u=x_{1}^{+}$after some rotation of the coordinate system.

Proof. By compactness (4.1) is valid for Lipschitz $\psi$. Choose

$$
\psi(r)= \begin{cases}1 & \text { if } r \leq e^{N} \\ 2-\frac{\log r}{N} & \text { if } e^{N}<r \leq e^{2 N} \\ 0 & \text { if } r>e^{2 N}\end{cases}
$$

Therefore we get from (4.1)

$$
\int_{B_{e^{N}} \cap \partial\{u>0\}} H \leq \widehat{C} \int_{B_{e^{2 N}}}|\nabla \psi|^{2}
$$




$$
=2 \pi \widehat{C} \int_{e^{N}}^{e^{2 N}} \frac{1}{r^{2} N^{2}} r d r=\frac{2 \pi \widehat{C}}{N} \rightarrow 0 \text { as } N \rightarrow \infty .
$$

Since $H=\kappa \geq 0$ it follows that $\partial\{u>0\}$ is a line $\ell$. After odd reflection of $u$ across $\ell$ we can apply Liouville's theorem to conclude that the reflected function is linear and hence the desired result follows.

\section{The Double CONE SOlution to $p$-LAPlacian in $\mathbb{R}^{3}$}

In this section we consider the case $F(t)=t^{p / 2}, 1<p<\infty$. The full regularity of minimizers in $\mathbb{R}^{2}$ is proved in [DP06].

7.1. $p$-Legendre equations. For $n=3$ and $x=\rho(\cos \phi \sin \theta, \sin \phi \sin \theta, \cos \theta)$ we put

$$
u=\rho \max \left(\frac{f(\theta)}{\dot{f}\left(\theta_{0}\right)}, 0\right),
$$

where $f$ is the solution of the differential equation

$$
\ddot{f}+(f+\cot \theta \dot{f}) \frac{f^{2}+\dot{f}^{2}}{f^{2}+(p-1) \dot{f}^{2}}+f=0,
$$

and $\theta_{0}$ is the only zero of $f$ in $(0, \pi / 2]$, cf. [AC81] 2.7 .

It is worth to point out that for $p=2(7.1)$ is the Legendre equation for $n=1$, [Kel67] page 127, and in this case $Q_{1}(x)=y(x)=1-\frac{x}{2} \ln \frac{1-x}{1+x}$.

Rewriting the divergence of a vectorfield $W=\left(W_{1}, W_{2}, W_{3}\right)$ in spherical coordinates [Kel67] page 183 (12), we get

$$
\operatorname{div} W=\frac{1}{\rho^{2} \sin \theta}\left\{\frac{\partial}{\partial \rho}\left[\rho^{2} \sin \theta W_{1}\right]+\frac{\partial}{\partial \phi}\left[\rho W_{2}\right]+\frac{\partial}{\partial \theta}\left[\rho \sin \theta W_{3}\right]\right\} .
$$

Moreover, $\nabla u=\left(u_{\rho}, \frac{1}{\rho \sin \theta} u_{\phi}, \frac{1}{\rho} u_{\theta}\right)$, [Kel67] page 181 (10), hence for $W=|\nabla u|^{p-2} \nabla u$ the above formula for divergence yields

$$
\begin{aligned}
\Delta_{p} u & =\operatorname{div} W \\
& =\frac{1}{\rho^{2} \sin \theta}\left\{\frac{\partial}{\partial \rho}\left[\rho^{2} \sin \theta|\nabla u|^{p-2} u_{\rho}\right]+\frac{\partial}{\partial \phi}\left[\frac{1}{\sin \theta}|\nabla u|^{p-2} u_{\phi}\right]+\frac{\partial}{\partial \theta}\left[\sin \theta|\nabla u|^{p-2} u_{\theta}\right]\right\} .
\end{aligned}
$$

Since $u$ does not depend on $\phi$ and is homogeneous function of degree 1 it follows that

$$
2 \sin \theta\left[f^{2}+\dot{f}^{2}\right]^{\frac{p-2}{2}} f+\frac{d}{d \theta}\left\{\sin \theta\left[f^{2}+\dot{f}^{2}\right]^{\frac{p-2}{2}} \dot{f}\right\}=0,
$$

or equivalently

$$
\ddot{f}\left(f^{2}+(p-1) \dot{f}^{2}\right)+f\left(2 f^{2}+p \dot{f}^{2}\right)+\cot \theta \dot{f}\left(f^{2}+\dot{f}^{2}\right)=0
$$

which is (7.1). Thus $\Delta_{p} u=0$ in $\{u>0\}$ and moreover $|\nabla u|=\lambda^{*}$ on $\partial\{u>0\} \backslash\{0\}$ with $(p-1)^{-\frac{1}{p}}=\lambda^{*}$. The free boundary of $u$ is a double cone, see Figure 2 .

When $p=2$ it is shown in [AC81] that $u$ is not a minimizer. Note that the zero set of $u$ has non-trivial Lebesgue density since the free boundary is a cone. 

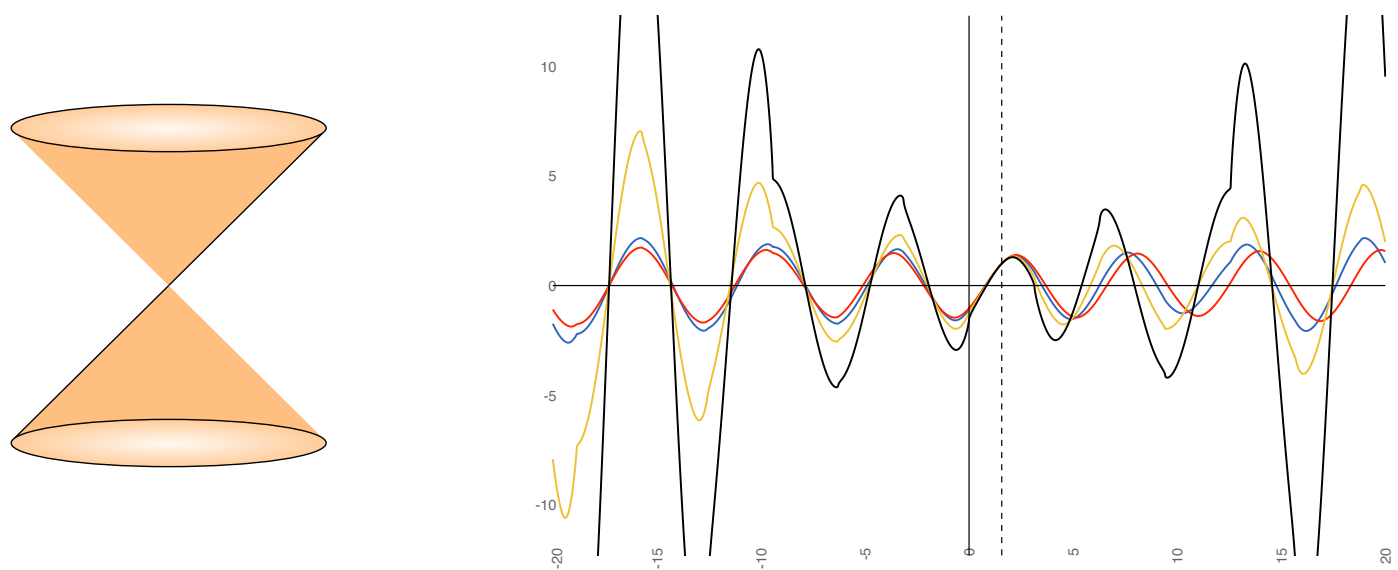

Figure 2. The double cone (left). The orange region is $\{u=0\}$. Some solutions of $(7.1)$ with $f(\pi / 2)=1, \dot{f}(\pi / 2)=1$ (right): black $p=4.3$, yellow $p=10$, blue $p=29$, red $p=100$. The dashed line is $x=\pi / 2$.

7.2. Reduction or 1st order ode. Let us introduce the function $u$ so that $\dot{f}=u f$ then substituting this into (7.1) we get the following first order equation for $u$

$$
\dot{u}+u^{2}+(1+u \cot \theta) \frac{1+u^{2}}{1+(p-1) u^{2}}+1=0 .
$$

In Figure 2 four solutions to (7.1) are illustrated for some values of $p$.

7.3. Stability inequality. The main result of this section is the following

Proposition 7.1. Let $u=\rho \max \left(\frac{f(\theta)}{\dot{f}\left(\theta_{0}\right)}, 0\right)$ where $f$ solves (7.1) and $\theta_{0}$ is the only zero of $f$ in $(0, \pi / 2]$. Then $u$ is not a minimizer.

Proof. Recall (5.4) and consider

$$
I=\int_{\{u>0\}}|\nabla u|^{p-2}\left\{|\nabla \psi|^{2}+(p-2) \frac{(\nabla u \nabla \psi)^{2}}{|\nabla u|^{2}}\right\}-\int_{\Gamma} H \psi^{2} .
$$

Note that

$$
I \leq(p-1) \int|\nabla u|^{p-2}|\nabla \psi|^{2}-\int_{\Gamma} H \psi^{2}
$$

Let us take the truncated fundamental solution

$$
\eta_{\delta}(x)= \begin{cases}\frac{1}{|x|} & \text { if }|x|>\delta, \\ \frac{1}{\delta} & \text { if }|x| \leq \delta,\end{cases}
$$

and consider $\psi_{R, \delta}(x)=\eta_{\delta}(x) \xi\left(\frac{|x|}{R}\right)$, where $0 \leq \xi \leq 1$ is a standard cut-off function such that $\xi(x)=1$ in $B_{1 / 2}, \xi \in C_{0}^{\infty}\left(B_{1}\right)$. 
Observe that

$$
\begin{aligned}
\lim _{R \rightarrow \infty} \int|\nabla u|^{p-2}\left|\nabla \psi_{R, \delta}\right|^{2} & =\int_{|x|>\delta}|\nabla u|^{p-2}\left|\nabla\left(\frac{1}{|x|}\right)\right|^{2} \\
& \leq C\|\nabla u\|_{\infty}^{p-2} \frac{1}{\delta}
\end{aligned}
$$

Denoting $\psi_{\delta}(x)=\eta_{\delta}(x)$ and using the divergence theorem we see that

$$
\begin{aligned}
\int|\nabla u|^{p-2}\left|\nabla \psi_{\delta}\right|^{2} & =\int_{\Gamma} \psi_{\delta} \partial_{\nu} \psi_{\delta}-\int \psi_{\delta} \operatorname{div}\left(|\nabla u|^{p-2} \nabla \psi_{\delta}\right) \\
& =\int_{\Gamma} \psi_{\delta} \partial_{\nu} \psi_{\delta}
\end{aligned}
$$

because

$$
\operatorname{div}\left(|\nabla u|^{p-2} \nabla\left(\frac{1}{|x|}\right)\right)=0, \quad \rho=|x|>\delta .
$$

We can see this from the equation

$$
\operatorname{div}\left(|\nabla u|^{p-2} \nabla\left(\frac{1}{|x|}\right)\right)=\frac{1}{\rho \sin \theta}\left\{\frac{\partial}{\partial \rho}\left(\rho^{2} \sin \theta g(\theta) \partial_{\theta} \psi_{\delta}\right)+\frac{\partial}{\partial \theta}\left(\rho \sin \theta \frac{1}{\rho} g(\theta) \partial_{\theta} \psi_{\delta}\right)\right\}
$$

where $g(\theta)=\left[f^{2}(\theta)+\dot{f}^{2}(\theta)\right]^{\frac{p-2}{2}}$. Since in $\rho>\delta$

$$
\frac{\partial}{\partial \rho}\left(\rho^{2} \sin \theta g(\theta) \partial_{\theta} \psi_{\delta}\right)=-\sin \theta g(\theta) \frac{\partial}{\partial \rho}\left(\rho^{2} \frac{1}{\rho^{2}}\right)=0
$$

and, moreover, $\partial_{\theta} \psi_{\delta}=0$ by definition of $\psi_{\delta}$, implying that

$$
\frac{\partial}{\partial \theta}\left(\rho \sin \theta \frac{1}{\rho} g(\theta) \partial_{\theta} \psi_{\delta}\right)=0
$$

Hence (7.4) is satisfied. Thus returning to (7.3) we conclude

$$
\begin{aligned}
I & \leq(p-1) \int_{\Gamma} \psi_{\delta} \partial_{\nu} \psi_{\delta}-\int_{\Gamma} H \psi_{\delta}^{2} \\
& =(p-1) \int_{\Gamma \cap\{|x|>\delta\}} \frac{1}{\rho^{2}} \frac{f}{\sqrt{f^{2}+(\dot{f})^{2}}}-\int_{\Gamma} H \psi_{\delta}^{2},
\end{aligned}
$$

because

$$
\begin{aligned}
\partial_{\nu} \psi_{\delta} & =-\frac{\nabla u}{|\nabla u|} \nabla \psi_{\delta}=-\frac{1}{\sqrt{f^{2}+\left(f^{\prime}\right)^{2}}}\left(-\frac{1}{\rho^{2}}, 0,0\right) \cdot(f, 0, \dot{f}) \\
& =\frac{1}{\rho^{2}} \frac{f}{\sqrt{f^{2}+(\dot{f})^{2}}} .
\end{aligned}
$$

But $\theta=\theta_{0}$ on $\Gamma \backslash B_{\delta}$ and $f\left(\theta_{0}\right)=0, \dot{f}\left(\theta_{0}\right) \neq 0$, so we have

$$
\frac{1}{\rho^{2}} \frac{f\left(\theta_{0}\right)}{\sqrt{f^{2}\left(\theta_{0}\right)+\left(\dot{f}\left(\theta_{0}\right)\right)^{2}}}=0
$$

Summarizing we infer

$$
I \leq-\int_{\Gamma} H \psi_{\delta}^{2}=-\int_{\delta}^{\infty} \frac{d \rho}{\rho^{2}} \int_{\mathbb{S}^{2} \cap \Gamma} \kappa<0
$$


where $\kappa>0$ is the curvature of the circle $\partial\{u>0\} \cap \partial B_{1}$.

\section{REFERENCES}

[All72] William K. Allard, On the first variation of a varifold, Ann. of Math. (2) 95 (1972), 417-491, DOI 10.2307/1970868. MR0307015 个5

[ATW93] Fred Almgren, Jean E. Taylor, and Lihe Wang, Curvature-driven flows: a variational approach, SIAM J. Control Optim. 31 (1993), no. 2, 387-438, DOI 10.1137/0331020. MR1205983 $\uparrow 4$

[AC81] H. W. Alt and L. A. Caffarelli, Existence and regularity for a minimum problem with free boundary, J. Reine Angew. Math. 325 (1981), 105-144. MR618549 ^2, 16

[ACF84] Hans Wilhelm Alt, Luis A. Caffarelli, and Avner Friedman, A free boundary problem for quasilinear elliptic equations, Ann. Scuola Norm. Sup. Pisa Cl. Sci. (4) 11 (1984), no. 1, 1-44. MR752578 个2, 3, 5, 6, 14

[BGM03] Elisabetta Barozzi, Eduardo Gonzalez, and Umberto Massari, The mean curvature of a Lipschitz continuous manifold, Atti Accad. Naz. Lincei Cl. Sci. Fis. Mat. Natur. Rend. Lincei (9) Mat. Appl. 14 (2003), no. 4, 257-277 (2004) (English, with English and Italian summaries). MR2104215 ^3, 4, 5

[CJK04] Luis A. Caffarelli, David Jerison, and Carlos E. Kenig, Global energy minimizers for free boundary problems and full regularity in three dimensions, Noncompact problems at the intersection of geometry, analysis, and topology, Contemp. Math., vol. 350, Amer. Math. Soc., Providence, RI, 2004, pp. 83-97, DOI 10.1090/conm/350/06339. MR2082392 $\uparrow 2,7$

[DTW12] Qiuyi Dai, Neil S. Trudinger, and Xu-Jia Wang, The mean curvature measure, J. Eur. Math. Soc. (JEMS) 14 (2012), no. 3, 779-800, DOI 10.4171/JEMS/318. MR2911884 $\uparrow 2,6$

[DP06] Donatella Danielli and Arshak Petrosyan, Full regularity of the free boundary in a Bernoulli-type problem in two dimensions, Math. Res. Lett. 13 (2006), no. 4, 667-681, DOI 10.4310/MRL.2006.v13.n4.a14. MR2250499 116

[DSJ09] Daniela De Silva and David Jerison, A singular energy minimizing free boundary, J. Reine Angew. Math. 635 (2009), 1-21, DOI 10.1515/CRELLE.2009.074. MR2572253 ^2

[DK18] Serena Dipierro and Aram L. Karakhanyan, Stratification of free boundary points for a two-phase variational problem, Adv. Math. 328 (2018), 40-81, DOI 10.1016/j.aim.2018.01.005. MR3771123 $\uparrow 15$

[Fel01] Mikhail Feldman, Regularity of Lipschitz free boundaries in two-phase problems for fully nonlinear elliptic equations, Indiana Univ. Math. J. 50 (2001), no. 3, 1171-1200, DOI 10.1512/iumj.2001.50.1921. MR1871352 115

[GT01] David Gilbarg and Neil S. Trudinger, Elliptic partial differential equations of second order, Classics in Mathematics, Springer-Verlag, Berlin, 2001. Reprint of the 1998 edition. MR1814364 $\uparrow 9$

[JS15] David Jerison and Ovidiu Savin, Some remarks on stability of cones for the one-phase free boundary problem, Geom. Funct. Anal. 25 (2015), no. 4, 1240-1257, DOI 10.1007/s00039-015-0335-6. MR3385632 $\uparrow 2$

[Kel67] Oliver Dimon Kellogg, Foundations of potential theory, Reprint from the first edition of 1929. Die Grundlehren der Mathematischen Wissenschaften, Band 31, Springer-Verlag, Berlin-New York, 1967. MR0222317 16

[O'N66] Barrett O'Neill, Elementary differential geometry, Academic Press, New York-London, 1966. MR0203595 $\uparrow 14$

[Sim83] Leon Simon, Lectures on geometric measure theory, Proceedings of the Centre for Mathematical Analysis, Australian National University, vol. 3, Australian National University, Centre for Mathematical Analysis, Canberra, 1983. MR756417 $\uparrow 5,6,7,15$

[Wei98] Georg S. Weiss, Partial regularity for weak solutions of an elliptic free boundary problem, Comm. Partial Differential Equations 23 (1998), no. 3-4, 439-455, DOI 10.1080/03605309808821352. MR1620644 ^2

Aram Karakhanyan: School of Mathematics, The University of Edinburgh, Peter Tait Guthrie RoAd, EH9 3FD Edinburgh, UK

E-mail address: aram6k@gmail.com 